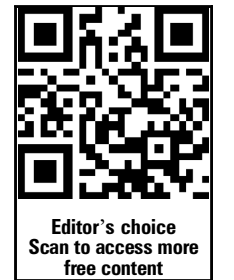

free content

'Department of Neurology, Stavanger University Hospital, Stavanger, Norway ${ }^{2}$ Department of Neurology, School of Medicine and Biomedical Sciences, Buffalo Neuroimaging Analysis Center, University at Buffalo, State University of New York, Buffalo, New York, USA ${ }^{3}$ Department of Clinical Medicine, University of Bergen, Bergen, Norway

${ }^{4} \mathrm{KG}$ Jebsen Centre for MS Research, Department of Clinical Medicine, University of Bergen, Bergen, Norway

${ }^{5}$ Department of Neurology, Norwegian Multiple Sclerosis Competence Centre, Haukeland University Hospital, Bergen, Norway

${ }^{6}$ Department of Radiology, Stavanger University Hospital, Stavanger, Norway ${ }^{7}$ MR Imaging Clinical Translational Research Center, School of Medicine and Biomedical Sciences, University at Buffalo, State University of New York, Buffalo, New York, USA

\section{Correspondence to} Dr Robert Zivadinov, Department of Neurology, School of Medicine and Biomedical Sciences, Buffalo Neuroimaging Analysis Center, 100 High St., Buffalo, NY 14203, USA; rzivadinov@bnac.net

Received 1 October 2013 Revised 15 January 2014 Accepted 26 January 2014 Published Online First 19 February 2014

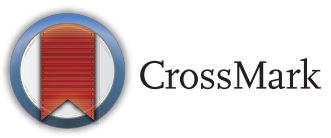

To cite: Jacobsen $C$ Hagemeier J, Myhr K-M, et al. I Neurol Neurosurg Psychiatry 2014;85: 1109-1115.

\title{
Brain atrophy and disability progression in multiple sclerosis patients: a 10-year follow-up study
}

\author{
Cecilie Jacobsen, ${ }^{1,2,3}$ Jesper Hagemeier, ${ }^{2}$ Kjell-Morten Myhr, ${ }^{4,5}$ Harald Nyland, ${ }^{4,5}$ \\ Kirsten Lode, ${ }^{1}$ Niels Bergsland, ${ }^{2}$ Deepa P Ramasamy, ${ }^{2}$ Turi 0 Dalaker ${ }^{3,6}$ \\ Jan Petter Larsen, ${ }^{3}$ Elisabeth Farbu, ${ }^{1,3}$ Robert Zivadinov ${ }^{2,7}$
}

\begin{abstract}
Objectives To identify MRI biomarkers associated with long-term disability progression in patients with multiple sclerosis (MS), and to define the rate of evolution of global, tissue-specific and regional atrophy in patients with MS over long-term.
\end{abstract}

Methods MRI of the brain and clinical neurological assessment was performed in 81 patients at time of first visit and after 5 and 10 years of follow-up. MRI was acquired on $1.5 \mathrm{~T}$ scanners. T1-lesion and T2-lesion volumes (LVs) were calculated. Global and tissue-specific atrophy changes were longitudinally assessed, using a direct measurement approach, by calculating percentage volume changes between different time points. Regional tissue volumes for the subcortical deep grey matter (SDGM) structures were also obtained. Disability progression was defined as an increase in Expanded Disability Status Scale of $\geq 1.0$ compared to baseline at 5 -year and 10-year follow-up.

Results Over 5 years, patients with disability progression showed significantly increased loss of whole brain $(-3.8 \%$ vs $-2.0 \%, p<0.001)$, cortical $(-3.4 \%$ vs $-1.8 \%, p=0.009)$ and putamen volume changes $(-10.6 \%$ vs $-3.8 \%, p=0.003)$ compared to patients with no disability progression. No significant change in white matter (WM) volume was observed when comparing progressing and non-progressing patients. Over 10 years, there was a trend for greater decrease in whole brain volume $(-5.5 \%$ vs $-3.7 \%, p=0.015)$ in the progressing patients. No significant changes in LV measures were detected between the patients with and without disability progression.

Conclusion This long-term study shows that whole brain, cortical and putamen atrophy occurs throughout the 10-year follow-up of this MS cohort and is more pronounced in the group that showed disability progression at 5, but not at 10 years of follow-up. Overall, GM atrophy showed better association with disease progression than WM atrophy over 5-year and 10-year follow-up.

\section{INTRODUCTION}

Multiple sclerosis (MS) is a chronic, inflammatory disease of the central nervous system characterised by multifocal disturbance of function. MS has, throughout the years, been viewed as a disease predominantly of the white matter (WM). However, during the past years, grey matter (GM) pathology has emerged as an important feature of the disease. Current conventional MRI techniques are limited in their ability to detect focal GM pathology because GM lesions have a different underlying pathology compared with WM lesions: they have minimal infiltration of immune cells and are of a less inflammatory nature. ${ }^{1-3}$ Also, the MRI visibility of GM lesions greatly depends on the size and location of the lesions. Newer imaging sequences such as double inversion recovery (DIR) have shown to detect substantially more cortical GM lesions than T2-weighted imaging (WI); however, still $80 \%$ of lesions seen histopathologically are missed using DIR. ${ }^{4}$ Ultra-high-field MRI has greatly improved detection of cortical GM lesions, but is not readily available. ${ }^{5}$

The search for reliable MRI markers to predict disease progression in MS has been ongoing for more than two decades. Standard MRI can be used to measure longitudinal changes in global, tissuespecific and regional brain volumes. GM atrophy has been found to correlate with disability progression in some studies, ${ }^{6-10}$ while others did not confirm this. ${ }^{11} 12$ The evolution of GM atrophy appears to commence early in the disease . $^{813-16}$ but the true rate of GM atrophy evolution over long-term is unknown at this time. Fisher $e t a^{17}$ showed a significant relationship between GM atrophy and disease progression over the 8-year follow-up. Several cross-sectional studies have shown an association between subcortical deep grey matter (SDGM) pathology and disability progression of MS. ${ }^{14}{ }^{18-20}$ However, further research to better understand temporal evolution of GM atrophy over long-term and its relation to disability progression is warranted and requires a long-term longitudinal study design, as recently proposed ${ }^{67}$

The main aim of the present study was to identify MRI biomarkers associated with disability progression in MS-patients after 5 and 10 years of follow-up. Another aim was to define the long-term rate of evolution of global, tissue-specific and regional atrophy in patients with MS.

\section{METHODS}

Patients

All patients diagnosed with MS during 1998 and 2000 in the county of Hordaland and southern parts of the county of Rogaland in south-western Norway were invited to participate in the study. Patients were included at the time of diagnosis. The diagnosis of MS was made according to the criteria of Poser. ${ }^{21} \mathrm{~A}$ total of 108 patients were identified; 
three of them had moved out of the study area, one had died and 11 refused to participate, leaving 93 patients. Out of those, 81 patients underwent full neurological assessment and MRI of the brain and were included in this prospective study (figure 1). Standardised clinical and MRI follow-up assessments took place after 5 and 10 years. This is an unselected cohort comprising of patients with all subgroups of MS disease, in a well-defined geographical area of Norway.

Information regarding medical history, demographic information and therapies was collected at baseline, 5-year and 10-year follow-up. MRI images and disability, as measured using the Kurtzke Expanded Disability Status Scale (EDSS), ${ }^{22}$ were also assessed at baseline, 5-year and 10-year follow-up. Written informed consent was obtained from all participants in accordance with the Helsinki Convention. The study has been approved by the Regional Committee for Medical and Health Research of Western Norway and The Norwegian Data Protection Authority.

\section{MRI acquisition and analysis}

MRI scans were performed in two different centres, at Haukeland University Hospital in Bergen and Stavanger University Hospital in Stavanger. The same standardised study protocol was used throughout the study. Scans were performed at $1.5 \mathrm{~T}$ (Siemens, Symphony/Phillips Medical systems, Intera) units. The MRI protocol consisted of a dual spin echo (SE) PD/T2-WI, a three-dimensional (3D) T1-WI and a SE T1-WI.

On the Philips scanner, all sequences were acquired with a $256 \times 256$ matrix, and a $230-\mathrm{mm}$ Field of View (FOV) for an in-plane resolution of $0.9 \times 0.9 \mathrm{~mm}^{2}$. Sequence-specific parameters were as follows: for the axial PD/T2, echo time (TE)1/ TE2/repetition time $(\mathrm{TR})=16 / / 80 \mathrm{~s} / 3240 \mathrm{~ms}$, flip angle (FA) $=90^{\circ}$, echo train length $(\mathrm{ETL})=6$, percent phase FOV $(\mathrm{pFOV})$ $=79.7$, slice thickness $=5 \mathrm{~mm}(\mathrm{gap}=1.5 \mathrm{~mm}), 2$ averages; for the axial 3D-T1: $\mathrm{TE} / \mathrm{TR}=4 / 17 \mathrm{~ms}, \mathrm{FA}=12^{\circ}$, $\mathrm{pFOV}=100$, slice thickness $=1.4 \mathrm{~mm}$ (no gap), 1 average; and for the axial SE T1, $\mathrm{TE} / \mathrm{TR}=12 / 525 \mathrm{~ms}, \mathrm{FA}=90^{\circ}, \mathrm{pFOV}=79.7$, slice thickness $=5$ $\mathrm{mm}$ (no gap), 1 average.

On the Siemens scanner, all sequences were acquired with a $256 \times 256$ matrix and a $230 \mathrm{~mm}$ (FOV) for an in-plane resolution of $0.9 \times 0.9 \mathrm{~mm}^{2}$. All acquisitions were upsampled on the console to a $512 \times 512$ matrix. Sequence-specific parameters were as follows: for the axial PD/T2 TE1/TE2/TR=13/81/ $3200 \mathrm{~ms}, \mathrm{ETL}=3, \mathrm{FA}=180^{\circ}, \mathrm{pFOV}=100$, slice thickness $=5 \mathrm{~mm}$ (no gap), 2 averages; for the sagittal 3D-T1, TE/TR $=10 / 25 \mathrm{~ms}$, $\mathrm{FA}=50^{\circ}, \mathrm{pFOV}=100$, slice thickness $=1.4 \mathrm{~mm}$ (no gap); and for the axial SE T1, TE $/ \mathrm{TR}=15 / 657 \mathrm{~ms}, \mathrm{FA}=90^{\circ}$, $\mathrm{pFOV}=100$, slice thickness $=5 \mathrm{~mm}$ (no gap), 2 averages.

Lesion volume

Using FMRIB's FLIRT (FMRIB's Linear Image Registration Tool), ${ }^{23}$ all baseline and follow-up images for a given subjects were co-registered to its baseline T1 SE image using a $6^{\circ}$ of freedom rigid-body model. All subsequent lesion analyses were done using the co-registered images. T2 and T1 lesion volumes (LVs) were calculated using a reliable semi-automated edge detection contouring/thresholding technique previously described. ${ }^{24}$ T1-LVs and T2-LVs in SDGM were also assessed at every time point.

Global and tissue-specific atrophy measures

Prior to using the 3D-T1 for subsequent analysis, it was modified using an in-house developed inpainting technique to avoid
Figure 1 Flow chart of drop-out reasons for discontinuation of the study of the multiple sclerosis patients included at baseline, 5-year and 10-year follow-up. RR, relapsing remitting; SP, secondary progressive; $\mathrm{PP}$, primary progressive.

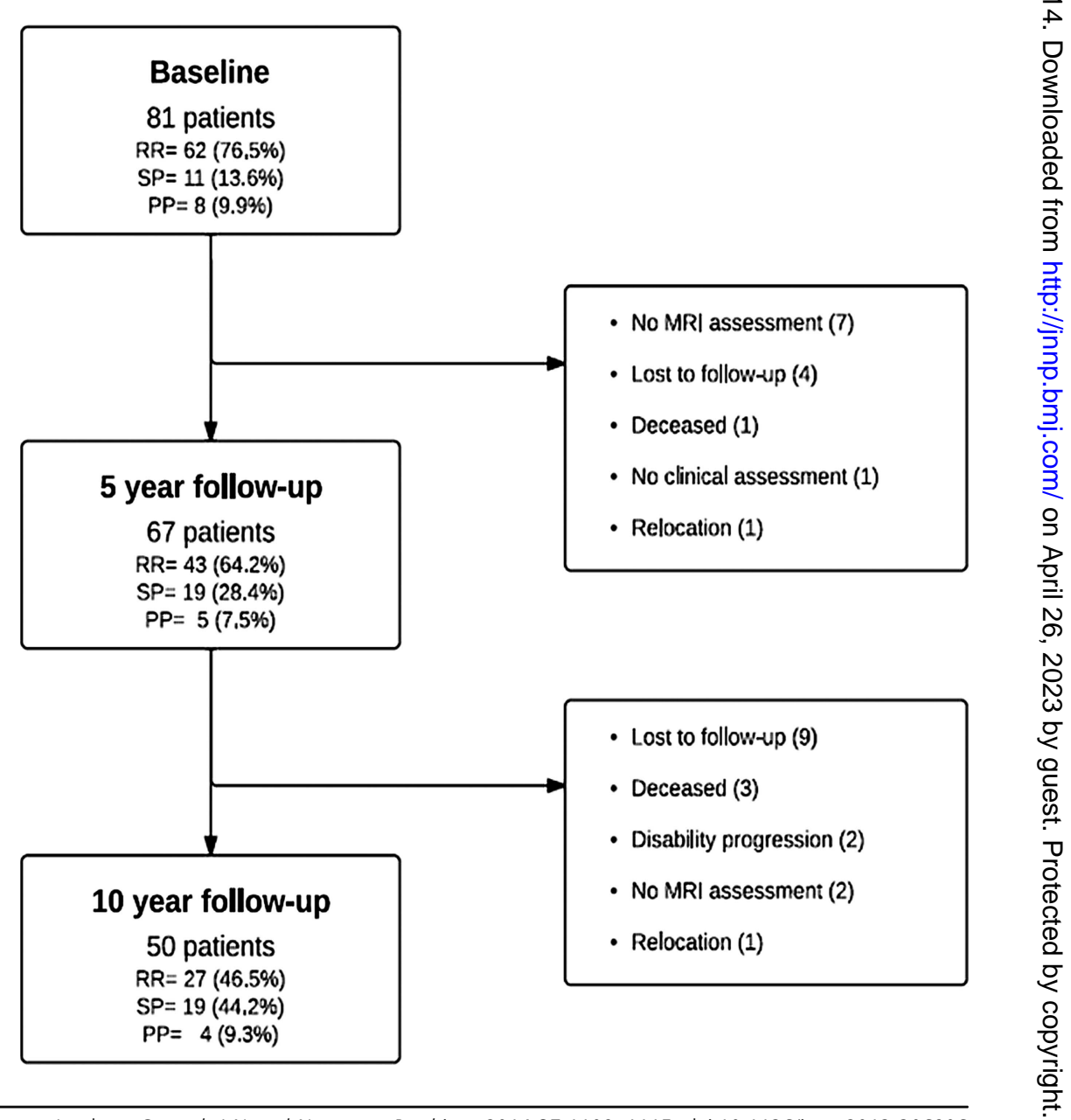


the impact of WM lesions on GM volume measurements. ${ }^{25}$ Normalised measures for whole brain volume (NWBV), normalised GM volume (NGMV), normalised white WM volume (NWMV), normalised cortical volume (NCV) and normalised lateral ventricle volume (LVV) were measured using SIENAX (V.2.6) as previously described. ${ }^{25} 26$

For longitudinal changes of the WBV, we applied the Structural Imaging Evaluation, with Normalisation, of Atrophy (SIENA) method to calculate the percentage brain volume change (PBVC). ${ }^{26}$ To quantify longitudinal GM, WM, cortical and lateral ventricle volume changes, we used a modified hybrid of FMRIB's SIENA and SIENAX tools, called SIENAX multi-timepoint (SX-MTP). ${ }^{27}$ Briefly, we used a brain-constrained and skullconstrained co-registration technique to place baseline and follow-up images into a joint space halfway between the two at all the time points in the study. Next, we combined baseline and follow-up intracranial volume masks via union, and valid voxel masks via intersection, ensuring that the same imaging volume was analysed at both time points. Finally, we segmented the resulting images with a modified longitudinal version (L-FAST) of FMRIB's Automated Segmentation Tool (FAST) tissue segmentation tool that uses a 4-dimensional joint hidden random Markov field to prevent misclassification between time points when longitudinal intensity changes are lacking (or minimal). ${ }^{28}$ Each tissue compartment percentage volume change between different time points was calculated from partial volume maps directly from the images. ${ }^{27}$

Absolute tissue volumes for the thalamus, caudate, putamen, globus pallidus, hippocampus, amygdala and nucleus accumbens at each time point were estimated from inpainted 3D-T1 images with FMRIB's Integrated Registration and Segmentation Tool (FIRST, V.1.2), a model-based segmentation/registration tool. ${ }^{29}$ Normalised SDGM volumes were obtained by multiplying the estimated volumes from FIRST by the volumetric scaling factor from SIENAX. ${ }^{25}$ Each regional structure percentage volume change between different time points was calculated between two cross-sectional scans.

\section{Statistical analysis}

Statistical analyses were performed using SPSS V.16 (SPSS Inc, Chicago, Illinois, USA). Disability progression was defined as an increase of EDSS by 1.0 point if baseline score was less than 6.0 and 0.5 if baseline score was 6.0 or higher. In this manner the patients were classified into stable and disability worsening groups on the follow-up. Baseline demographic and MRI parameter comparisons were performed using Student $t$ test, $\chi^{2}$-test and Mann-Whitney U test, where appropriate. Furthermore, changes in longitudinal MRI measurements between time points (baseline to 5 years and baseline to 10 years) were compared between groups. The analyses of the 10 -year cohort $(n=50)$ were performed also for the 5 -year follow-up. Cohen's d-values were calculated to designate the effect sizes, where appropriate.

To assess whether change over time of MRI parameters was associated with disability progression at 5 and 10 years, binary logistic regression analysis was carried out. Absence or presence of disability progression at 5 and 10 years was the dependent variable. Age, sex, MS subtype at baseline, scanner type, disease duration and use of disease modifying treatment (DMT) were entered as covariates. We considered $\mathrm{p}$ values of $<0.01$ as significant and $<0.05$ as a trend, in order to minimise spurious findings, due to multiple comparisons.

\section{RESULTS}

Demographic, clinical and MRI characteristics at baseline:

Eighty-one patients were included and underwent MRI of the brain and clinical neurological examination at baseline. At 5-year and 10-year follow-up, 66 and 50 patients were still participating, respectively. Reasons for discontinuation over 5-year and 10-year follow-up are listed in figure 1. Mean months from symptom debut to diagnosis was 70.4 (SD 85.8). Mean years since diagnosis at baseline was 2.5 (SD 1.0).

Table 1 presents the baseline characteristics of the enrolled patient cohort. No significant differences between disability progressing and non-progressing groups at 5-year and 10-year follow-up were found in regard to baseline demographic data, disability status, MS subtype, presence of DMT and months on DMT. Comparisons of the groups included at baseline and the groups completing the 5-year and 10-year follow-up showed no significant differences in demographic, clinical or MRI characteristics at baseline.

In the first 5 years of the study 26 patients (39\%), and at 10 -year follow-up 25 patients (50\%), showed disability progression. The group as a whole worsened in terms of disability. Median baseline EDSS was 3.5 (IQR1.5) and increased to 3.5 (IQR 2.5) at 5-year follow-up, and further to 3.8 (IQR 3.5) at 10 -year follow-up. At baseline $13 \%$ of the patients were on DMT, at 5-year follow-up $41 \%$ in the disability progressing and $53 \%$ in the non-progressing groups were on DMT. At 10 -year follow-up $60 \%$ of the patients in both groups were on DMT.

Table 2 presents the baseline MRI characteristics of the enrolled patient cohort. No significant differences between disability progressing and non-progressing groups at 5-year and 10-year follow-up were found in regards to baseline MRI measures.

\section{Five-year follow-up}

In the first 5 years of the study, the group that showed disability progression had a decrease in WBV $(-3.8 \%$ vs $-2.0 \%$, $\mathrm{p}<0.001)$, cortical volume $(-3.4 \%$ vs $-1.8 \%, \mathrm{p}=.009)$ and in

Table 1 Demographics and clinical characteristics at baseline, and split by disability progression status at 5 and 10 -year follow-up

\begin{tabular}{|c|c|c|c|}
\hline & $\begin{array}{l}\text { Baseline } \\
n=81\end{array}$ & $\begin{array}{l}\text { 5-year } \\
\text { follow-up } \\
n=67\end{array}$ & $\begin{array}{l}\text { 10-year } \\
\text { follow-up } \\
n=50\end{array}$ \\
\hline Gender, female, n (\%) & $55(67.1)$ & $45(67.2)$ & $33(66)$ \\
\hline Age in years, mean (SD) & $42(9.5)$ & $41(9.5)$ & $41(9)$ \\
\hline $\begin{array}{l}\text { Disease duration in months, } \\
\text { mean (SD) }\end{array}$ & $100(88)$ & $101(96)$ & $105(100)$ \\
\hline $\begin{array}{l}\text { EDSS, } \\
\text { median (range), IQR }\end{array}$ & $\begin{array}{l}3.5(0-6.0) \\
1.5\end{array}$ & $\begin{array}{l}3.5(0-6.0) \\
1.5\end{array}$ & $\begin{array}{l}3.5(0-6.0) \\
1.5\end{array}$ \\
\hline \multicolumn{4}{|l|}{ Disease course, n (\%) } \\
\hline RRMS & $62(76.5)$ & $55(82.1)$ & $43(86)$ \\
\hline SPMS & $11(13.6)$ & $8(11.9)$ & $4(8)$ \\
\hline PPMS & $8(9.9)$ & $4(6)$ & $3(6)$ \\
\hline Patients on DMT n (\%) & $11(13.4)$ & $9(13.4)$ & $8(16.3)$ \\
\hline \multicolumn{4}{|l|}{ DMT type, n (\%) } \\
\hline None & 70 (86.6) & 58 (86.6) & $42(84)$ \\
\hline Interferon $\beta$ & $9(11)$ & $8(11.9)$ & 7 (14) \\
\hline Glatiramer acetate & $2(2.4)$ & $1(1.5)$ & $1(4)$ \\
\hline Mitoxantrone & 0 & 0 & 0 \\
\hline Natalizumab & 0 & 0 & 0 \\
\hline Other & 0 & 0 & 0 \\
\hline
\end{tabular}

Disability progression was measured using the EDSS. The differences in demographics between the disability progressing and non-progressing groups were calculated using $\chi^{2}$, Student $t$ test and Mann-Whitney rank sum tests, as appropriate.

DMT, disease modifying treatment; EDSS, Expanded Disability Status Scale; MS, multiple sclerosis; PP, primary progressive; $R R$, relapsing remitting; $S P$, secondary progressive. 
Table 2 Baseline MRI brain characteristics split by disability progression status at 5-year and 10-year follow-up

\begin{tabular}{|c|c|c|c|c|c|c|}
\hline & \multicolumn{2}{|c|}{$\begin{array}{l}\text { 5-year follow-up split by disability } \\
\text { progression }\end{array}$} & \multirow[b]{2}{*}{$\mathrm{p}$ Value } & \multicolumn{2}{|c|}{$\begin{array}{l}\text { 10-year follow-up split by disability } \\
\text { progression }\end{array}$} & \multirow[b]{2}{*}{ p Value } \\
\hline & Yes $(n=27)$ & No $(n=40)$ & & Yes $(n=25)$ & No $(n=25)$ & \\
\hline T2 lesion volume & $19.22(15.62)[16.18]$ & $10.91(10.82)[7.82]$ & ns & $16.75(14.97)[13,15]$ & $9.88(10.53)[7.30]$ & ns \\
\hline T1 lesion volume & $7.07(6.85)[6.85]$ & $3.74(5.23)[2.33]$ & * & $5.09(4.87)[3.27]$ & $2.89(4.29)[2.15]$ & ns \\
\hline Normalised whole brain volume & $1.46(0.076)[1.47]$ & $1.48(0.09)[1.46]$ & ns & $1.46(0.08)[1.47]$ & $1.49(0.08)[1.49]$ & ns \\
\hline Normalised grey matter volume & $0.75(0.05)[0.75]$ & $0.77(0.06)[0.76]$ & ns & $0.75(0.05)[0.75]$ & $0.77(0.06)[0.77]$ & ns \\
\hline Normalised white matter volume & $0.71(0.04)[0.70]$ & $0.71(0.05)[0.71]$ & ns & $0.71(0.04)[0.70]$ & $0.72(0.04)[0.72]$ & ns \\
\hline Normalised lateral ventricular volume & $0.06(0.02)[0.05]$ & $0.05(0.02)[0.05]$ & ns & $0.06(0.02)[0.05]$ & $0.05(0.02)[0.05]$ & ns \\
\hline Normalised cortical volume & $0.57(0.04)[0.56]$ & $0.58(0.05)[0.58]$ & ns & $0.57(0.04)[0.56]$ & $0.59(0.05)[0.59]$ & ns \\
\hline Normalised total SDGM volume & $0.04(0.01)[0.04]$ & $0.05(0.01)[0.05]$ & ns & $0.04(0.01)[0.04]$ & $0.05(0.01)[0.05]$ & ns \\
\hline \multicolumn{7}{|l|}{ Subcortical deep grey matter structures } \\
\hline T2 lesion SDGM volume & $0.25(0.34)[0.12]$ & $0.14(0.30)[0.04]$ & ns & $0.17(0.27)[0.02]$ & $0.16(0.33)[0.05]$ & ns \\
\hline T1 lesion SDGM volume & $0.05(0.09)[0]$ & $0.03(0.06)[0]$ & ns & $0.03(0.05)[0]$ & $0.03(0.06)[0]$ & ns \\
\hline Caudate & $6.39(0.98)[5.96]$ & $6.30(0.87)[6.10]$ & ns & $6.12(0.91)$ [5.91] & $6.35(0.94)$ [6.25] & ns \\
\hline Putamen & $9.21(1.19)[9.48]$ & $9.33(1.43)[9.10]$ & ns & $9.19(1.07)$ [9.48] & $9.40(1.36)[9.16]$ & ns \\
\hline Globus pallidus & $3.35(0.51)$ [3.29] & $3.46(0.42)[3.37]$ & ns & $3.41(0.44)[3.48]$ & $3.49(0.42)$ [3.41] & ns \\
\hline Thalamus & $14.45(1.82)$ [14.38] & $15.06(1.85)$ [15.23] & ns & $14.63(1.84)$ [15.04] & 15.37 (1.72) [15.35] & ns \\
\hline Hippocampus & $7.20(0.82)$ [7.23] & 7.63 (1.26) [7.55] & ns & $7.18(0.96)$ [7.23] & $7.83(1.17)$ [7.82] & * \\
\hline Amygdala & $2.74(0.48)[2.78]$ & $2.80(0.45)[2.79]$ & ns & $2.75(0.44)[2.71]$ & $2.88(0.44)[2.83]$ & ns \\
\hline Accumbens & $0.88(0.22)[0.85]$ & $0.88(0.23)[0.90]$ & ns & $0.83(0.20)[.84]$ & $0.92(0.23)[0.94]$ & ns \\
\hline
\end{tabular}

Disability progression was measured using the Expanded Disability Status Scale.

The brain volume values are presented in litres, lesion volumes and SDGM volumes in millilitres, as mean, (SD), [median]. The differences between the disability progressing and non-progressing groups were calculated using Student $t$ test and Mann-Whitney rank sum test, as appropriate.

*Trend at $p<0.05$.

ns, non-significant; SDGM, subcortical deep grey matter.

putamen volume $(-10.6 \%$ vs $-3.8 \%, \mathrm{p}=.003)$ (table 3$)$. The disability-progressing and non-progressing patients exhibited no significant changes in global or regional SDGM lesion measures over the 5-year follow-up, although total SDGM, globus pallidus and amygdala volume loss showed a trend for being greater in the disability progressing MS patients. T1-LVs and T2-LVs were largely unchanged in both groups.

In logistic regression analysis, corrected for baseline volumes of the specific structure, T1-LVs and T2-LVs, age, sex, scanner type, MS subtype and months on DMT, showed that decreased WBV $(\beta=-0.571, p=0.002)$ and cortical volume $(\beta=-0.338$, $\mathrm{p}=0.009$ ) were significantly associated with disability progression (table 5).

\section{Ten-year follow-up}

At the 10-year follow-up, none of the brain volume measures differed in the disability-progressing and non-progressing groups (table 4). Decrease in WBV showed only a trend for being greater in the group showing disability progression $(-5.54 \%$ vs $-3.73 \%, p=0.015)$, although there was a significant difference at 5 -year follow-up $(p=0.009$, table 4). The disability-progressing and non-progressing patients exhibited no changes in global or regional SDGM lesion measures, T1-LVs or T2-LVs over the 5-year follow-up.

Similar logistic regression analysis, corrected for baseline volumes of the specific structure, T1-LVs and T2-LVs, age, sex, scanner type, MS subtype and months on DMT, showed a trend for association between decrease in WBV and disability progression $(\beta=-0.391, p=0.02)$ after 10 years (table 5$)$.

No significant differences with respect to type of treatment, duration of treatment and changes of treatment status were
Table 3 Changes in MRI volumes between baseline and 5-year follow-up

\begin{tabular}{|c|c|c|c|c|}
\hline & \multicolumn{2}{|c|}{$\begin{array}{l}\text { At } 5 \text {-year follow-up split by } \\
\text { disability progression }\end{array}$} & \multirow[b]{2}{*}{ Cohen's d } & \multirow[b]{2}{*}{$\mathrm{p}$ Value } \\
\hline & Yes $(n=27)$ & No $(n=40)$ & & \\
\hline T2 lesion volume & $5.88(10.81)$ & $2.76(7.89)$ & 0.33 & ns \\
\hline T1 lesion volume & $3.83(7.50)$ & $1.89(4.20)$ & 0.32 & ns \\
\hline Whole brain volume & $-3.75(2.21)$ & $-1.98(1.29)$ & 0.98 & ** \\
\hline Grey matter volume & $-3.00(3.49)$ & $-2.05(3.12)$ & 0.29 & ns \\
\hline White matter volume & $-1.081(4.45)$ & $-1.10(2.92)$ & 0.01 & ns \\
\hline $\begin{array}{l}\text { Lateral ventricular } \\
\text { volume }\end{array}$ & $20.03(23.16)$ & $11.03(16.35)$ & 0.45 & ns \\
\hline Cortical volume & $-3.44(2.89)$ & $-1.83(1.95)$ & 0.65 & ** \\
\hline Total SDGM volume & $-6.60(6.70)$ & $-2.96(4.54)$ & 0.64 & * \\
\hline \multicolumn{5}{|c|}{ Subcortical deep grey matter structures } \\
\hline $\mathrm{T} 2$ lesion SDGM volume & $18.35(401.4)$ & $-26.8(373.5)$ & 0.12 & ns \\
\hline T1 lesion SDGM volume & $2.48(147.4)$ & $0.25(73.9)$ & 0.02 & ns \\
\hline Caudate & $-6.05(6.42)$ & $-3.26(5.53)$ & 0.47 & ns \\
\hline Putamen & $-10.56(11.70)$ & $-3.81(6.50)$ & 0.71 & ** \\
\hline Globus pallidus & $-6.67(10.21)$ & $-1.74(6.46)$ & 0.58 & * \\
\hline Thalamus & $-5.04(6.19)$ & $-2.73(4.29)$ & 0.43 & ns \\
\hline Hippocampus & $-4.06(7.49)$ & $-2.61(5.82)$ & 0.22 & ns \\
\hline Amygdala & $-5.37(12.34)$ & $-0.38(7.49)$ & 0.49 & * \\
\hline Accumbens & $-15.08(19.05)$ & $-9.11(12.68)$ & 0.36 & ns \\
\hline
\end{tabular}

Disability progression was measured using the Expanded Disability Status Scale. Lesion volume changes are expressed in absolute change and SDs in millilitres. All brain volume changes are expressed in percentage changes and SDs.

The differences between the disability progressing and non-progressing groups were calculated using Student t test and Mann-Whitney rank sum test, as appropriate.

** Significant at $p<0.01$.

${ }^{*}$ Trend at $\mathrm{p}<0.05$

ns, non-significant; SDGM, subcortical deep grey matter. 
Table 4 Changes in MRI characteristics between baseline and 5-year and 10-year follow-up for the group followed over 10 years

\begin{tabular}{|c|c|c|c|c|c|c|c|c|}
\hline & \multicolumn{2}{|c|}{$\begin{array}{l}\text { 5-year follow-up split by } \\
\text { disability progression }\end{array}$} & \multirow[b]{2}{*}{ Cohen's d } & \multirow[b]{2}{*}{ p Value } & \multicolumn{2}{|c|}{$\begin{array}{l}\text { 10-year follow-up split by } \\
\text { disability progression }\end{array}$} & \multirow[b]{2}{*}{ Cohen's d } & \multirow[b]{2}{*}{ p Value } \\
\hline & Yes $(n=18)$ & No $(n=32)$ & & & Yes $(n=25)$ & No $(n=25)$ & & \\
\hline T2 lesion volume & $2.15(7.03)$ & $2.07(6.49)$ & 0.01 & ns & $4.05(8.79)$ & $4.87(7.03)$ & 0.10 & ns \\
\hline T1 lesion volume & $1.84(3.98)$ & 930 & 0.03 & ns & $1.53(3.73)$ & $1.14(3.74)$ & 0.10 & ns \\
\hline Whole brain volume & $-3.24(2.21)$ & $-1.92(1.26)$ & 0.73 & * & $-5.54(2.69)$ & $-3.73(1.88)$ & 0.78 & ** \\
\hline Grey matter volume & $-2.60(3.04)$ & $-2.41(2.67)$ & 0.07 & ns & $-4.96(3.23)$ & $-5.14(3.55)$ & 0.05 & ns \\
\hline White matter volume & $-1.45(2.35)$ & $-1.45(2.81)$ & 0 & ns & $-0.711(2.69)$ & $-0.73(4.30)$ & 0 & ns \\
\hline Lateral ventricular volume & $15.60(15.67)$ & $12.74(13.29)$ & 0.20 & ns & $28.16(22.41)$ & $22.27(48.96)$ & 0.15 & ns \\
\hline Cortical volume & $-2.70(2.75)$ & $-1.89(1.95)$ & 0.34 & ns & $-4.00(3.24)$ & $-3.21(2.97)$ & 0.25 & ns \\
\hline Total SDGM volume & $-5.33(4.97)$ & $-2.74(3.52)$ & 0.60 & ns & $-7.28(7.34)$ & $-5.07(4.31)$ & 0.37 & ns \\
\hline \multicolumn{9}{|c|}{ Subcortical deep grey matter structures } \\
\hline T2 lesion SDGM volume & $3.08(375.6)$ & $-12.33(372.7)$ & 0 & ns & $-2.50(306.8)$ & $-25.63(213.4)$ & 0 & ns \\
\hline T1 lesion SDGM volume & $12.67(133.3)$ & $8.34(73.8)$ & 0.04 & ns & 7.75 (109.6) & $-3.83(89)$ & 0.04 & ns \\
\hline Caudate & $-5.20(5.12)$ & $-2.88(4.98)$ & 0.46 & ns & $-6.69(6.62)$ & $-7.58(5.84)$ & 0.14 & ns \\
\hline Putamen & $-8.10(7.06)$ & $-3.78(6.04)$ & 0.66 & ** & $-11.17(13.12)$ & $-5.91(5.87)$ & 0.52 & ns \\
\hline Globus pallidus & $-5.06(7.83)$ & $-0.80(5.33)$ & 0.64 & ** & $-6.51(11.15)$ & $-2.91(6.38)$ & 0.40 & ns \\
\hline Thalamus & $-4.23(4.63)$ & $-2.72(3.58)$ & 0.36 & ns & $-5.83(5.94)$ & $-4.33(4.41)$ & 0.29 & ns \\
\hline Hippocampus & $-3.06(6.95)$ & $-2.20(4.84)$ & 0.14 & ns & $-6.44(9.31)$ & $-4.69(4.44)$ & 0.24 & ns \\
\hline Amygdala & $-4.32(10.90)$ & $-0.24(5.71)$ & 0.47 & ns & $-2.07(9.88)$ & $-1.23(7.38)$ & 0.10 & ns \\
\hline Accumbens & $-12.77(19.12)$ & $-9.75(13.10)$ & 0.18 & ns & $-14.34(18.96)$ & $-13.07(12.39)$ & 0.08 & ns \\
\hline
\end{tabular}

detected on lesion, global, tissue-specific and regional brain volume measures over 5-year and 10-year follow-up.

\section{DISCUSSION}

In this prospective study of a cohort of MS patients included at time of diagnosis in our centres, we investigated the association between MRI measures of global and tissue-specific damage and disability evolution, as measured by EDSS.

The main findings of the study are that the development of whole brain, cortical and putamen atrophy was associated with disability progression 5 years after MS diagnosis. Whole brain atrophy showed only a trend for an association with disability progression 10 years after diagnosis, but none of the other MRI parameters were associated with disability progression.

MS has for many years been regarded as a disease of the cerebral WM and spinal cord. ${ }^{30} 31$ Subsequently diagnostic and therapeutic approaches were aimed accordingly. However, volumetric brain tissue analysis has shown GM pathology is an important feature of the disease. The emergence of advanced MRI techniques in the past decade has further confirmed that the involvement of GM. ${ }^{30-32}$ MS-related brain atrophy has been reported in most brain structures. ${ }^{6} 7910$ 12-17 33-35 A majority of the studies have found atrophy to be greater in the GM, ${ }^{6} 79$ 1012173334 others reported equal involvement of the GM and $\mathrm{WM}^{36}$ while some studies reported predominantly WM atrophy. ${ }^{37}$ It may be that discrepancy between the study findings is in part due to lack of having performed lesion filling prior to segmentation. WM hypointensities are known to negatively influence the results of automated segmentation techniques and yield incorrect tissue segmentations. ${ }^{38} \mathrm{GM}$ atrophy has been detected early in the disease course and the present study findings support the growing evidence of importance of GM atrophy in MS.? ${ }^{73-163335}$
In the present cohort, loss in WBV during 5 and 10 years was driven by a greater decline in GM than WM volume. Cortical and putamen volume loss were significantly associated with disability progression after 5 years, but the WM volume was not. This is in line with a recent longitudinal study showing that cortical atrophy and thalamus atrophy in relapsing remitting multiple sclerosis (RRMS) were significantly associated with

Table 5 Logistic regression analysis of MRI variables associated with disability progression at 5-year and 10-year follow-up

\begin{tabular}{|c|c|c|c|c|}
\hline & \multicolumn{2}{|c|}{ 5-year follow-up } & \multicolumn{2}{|c|}{ 10-year follow-up } \\
\hline & $\boldsymbol{\beta}$ & $\mathrm{p}$ Value & $\boldsymbol{\beta}$ & $p$ Value \\
\hline Whole brain volume & -0.571 & ** & -0.391 & * \\
\hline Grey matter volume & -0.124 & ns & 0.017 & ns \\
\hline White matter volume & 0.049 & ns & -0.004 & ns \\
\hline Lateral ventricular volume & 0.018 & ns & 0.006 & ns \\
\hline Cortical volume & -0.338 & ** & -0.126 & ns \\
\hline Total SDGM volume & -0.113 & * & -0.072 & ns \\
\hline \multicolumn{5}{|c|}{ Subcortical deep grey matter structures } \\
\hline Caudate & -0.114 & * & 0.26 & ns \\
\hline Putamen & -0.078 & * & -0.073 & ns \\
\hline Pallidus & -0.086 & * & -0.055 & ns \\
\hline Thalamus & -0.070 & ns & -0.065 & ns \\
\hline Hippocampus & -0.025 & ns & -0.039 & ns \\
\hline Amygdala & -0.072 & * & -0.016 & ns \\
\hline Accumbens & -0.002 & ns & 0.000 & ns \\
\hline
\end{tabular}

Logistic regression analysis corrected for age, sex, scanner type, baseline disease duration, T1-LVs and T2-LVs, MS subtype and months and type of DMT. Disability progression was measured using the Expanded Disability Status Scale. ** Significant at $p<0.01$.

${ }^{*}$ Trend at $p<0.05$. 
disability progression over 5 years. ${ }^{6}$ Similar to our findings, they also showed that WM volume was largely unchanged during the first 5 years. ${ }^{6} 31$ These findings strengthen the increasing evidence $^{8} 10192039$ that GM pathology may be playing a crucial role in MS-related disability progression. ${ }^{40}$ This was also confirmed by the logistic regression analysis showing that the rate of WB and cortical atrophy were the only MRI measures independently associated with disability status at the 5-year follow-up, adjusted for age, sex, scanner type, disease course, baseline volumes of T1-lesion and T2-lesion at baseline, baseline volume of the specific brain structures, as well as months on DMT.

Some studies reported that patients having greater atrophy at baseline were more likely to develop disability progression over time. ${ }^{10} 1839{ }^{41}$ However, in our cohort, baseline volume measures were not associated with disability progression after 5 and 10 years.

There are several possible reasons why a correlation between atrophy rate and disability progression was not detected at the 10-year follow-up. A decreased atrophy rate over time related to natural history has been suggested, with a faster rate in the first few years of disease than later in the disease course. ${ }^{42}$ We included patients diagnosed during 1998 and 2000 and defined baseline by the MRI at the time of inclusion. Thus, disease duration at baseline was quite long and varied between the patients (mean 100, median 60 months). This was due to the interval between diagnosis and inclusion and the interval between the first symptom and diagnosis. The latter might relate to use of the Poser criteria ${ }^{21}$ at the time of patient enrolment, which implied that a definite MS diagnosis was probably made at a later point in the disease course, than it would have been by applying the current used revised McDonald diagnostic criteria. $^{43}$

A majority of the patients in the current study were on DMT at the 10-year follow-up, and some studies have shown decreased atrophy rate with DMT use. ${ }^{30}{ }^{41}$ However, no significant difference in the atrophy rate was found between treated and non-treated patients at 5-year and 10-year follow-up in this prospective cohort.

In line with some previous studies, although not significantly related, baseline T1-LV showed a stronger association with disability progression at the 5 -year follow-up than the T2-LV. ${ }^{44} \mathrm{~A}$ recent study by Popescu et al. ${ }^{45}$ showed central atrophy and LV change to significantly predict EDSS in a large group of MS patients. In the present study, we found that increased T1-LV at baseline showed a trend for the greater disability progression over 5 years; however, no association was found with T2-LV and SDGM-LV over the follow-up.

There are a number of limitations in this study that need to be considered. First, our sample size was relatively small and there was a considerable drop-out (31 patients, 38.3\%) from baseline until the 10-year follow-up. However, comparisons of the baseline characteristics the groups completing the 5-year and 10-year follow-up showed no significant differences in demographic, clinical or MRI characteristics at baseline. Second, the MRI data were acquired from two different scanners, but the effects of the different scanners on detection of longitudinal volume changes are rather small. ${ }^{46}$ To account for this issue, we used scanner type, as covariate, in analyses. Informative censoring could be another possible reason as 4 patients deceased, 2 patients discontinued the study because of disability progression, and 12 patients discontinued for unknown reasons. This may have contributed to the MRI measurement results at the 10-year follow-up, as these subjects could have been those that atrophy or lesion burden outcomes progressed the most. While we adjusted for baseline MRI differences of the groups completing the 5 -year and 10-year follow-up, it is possible that the patients who dropped out of the study deteriorated more quickly. The lack of healthy control comparison group in the study is also a weakness. For this reason any comparison of baseline versus follow-up atrophy rate in MS patients versus healthy population is difficult to interpret. Finally, the disability progression was assessed at 5 and 10 years at the single time point, while evaluation of 3-month or 6-month sustained disability progression would have been preferred.

In conclusion, this prospective study showed that WB, cortical and SDGM atrophy occurs in the course of MS and are associated with disability progression at 5 , but not at 10 years of the follow-up. Over 10 years, WB atrophy showed better association with disability progression than tissue-specific or regional GM measures. Overall, GM atrophy showed a stronger association with disease progression than WM atrophy over 5-year and 10-year follow-up.

The search for biomarkers that can predict the course of MS is an ongoing pursuit. MRI is one of the available tools to monitor disease activity, but is well known not to correlate very well with clinical outcomes. Assessment of GM pathology may add to the understanding of the disease progression in MS, and overcome the clinical-MRI paradox.

Contributors $\mathrm{CJ}$ and RZ participated in the study concept and design, data analysis and the manuscript preparation. $\mathrm{JH}$ participated in data analysis and revised the manuscript critically for important intellectual content. NB and DPR performed MRI data analysis and revised the manuscript critically for important intellectual content. All other authors participated on study concept and design and revised the manuscript critically for important intellectual content.

Competing interests $\mathrm{NB}, \mathrm{DPR}, \mathrm{HN}, \mathrm{KL}$ and $\mathrm{JH}$ have nothing to disclose. $\mathrm{CJ}$ was awarded a neuroimaging fellowship with BNAC. MK-M has received honoraria for lecturing, participation in advisory boards or pharmaceutical company sponsored clinical trials, and travel support from Allergan, Almirall, Bayer Schering, Biogen Idec, Genzyme, Merck-Serono, Novartis, Sanofi Aventis and/or Teva. JPL has participated in advisory boards for Lundbeck Pharma and GSK. TODalaker has received a grant and honoraria for a lecture from Biogen Idec. EF received an unrestricted research grant from Novartis, participated in advisory boards, received speaker honoraria and travel support from Biogen Idec, Genzyme, Novartis, Merck Serono, Teva, Almirall and Bayer. RZ received personal compensation from Biogen Idec, EMD Serono, Novartis, Claret and Sanofi-Genzyme for speaking and consultant fees. RZ received financial support for research activities from Biogen Idec, Teva Pharmaceuticals, EMD Serono, Novartis and Sanofi-Genzyme.

Ethics approval The Regional Committee for Medical and Health Research of Western Norway and The Norwegian Data Protection Authority.

Provenance and peer review Not commissioned; externally peer reviewed.

\section{REFERENCES}

1 Bo L, Geurts JJ, Mork SJ, et al. Grey matter pathology in multiple sclerosis. Acta Neurol Scand Supp/ 2006;183:48-50.

2 Hulst HE, Geurts JJ. Gray matter imaging in multiple sclerosis: what have we learned? BMC Neurol 2011;11:153.

3 Popescu BF, Lucchinetti CF. Meningeal and cortical grey matter pathology in multiple sclerosis. BMC Neurol 2012;12:11.

4 Seewann A, Kooi EJ, Roosendaal SD, et al. Postmortem verification of MS cortical lesion detection with 3D DIR. Neurology 2012;78:302-8.

5 Geurts JJ, Calabrese M, Fisher E, et al. Measurement and clinical effect of grey matter pathology in multiple sclerosis. Lancet Neurol 2012;11:1082-92.

6 Zivadinov R, Bergsland N, Dolezal 0, et al. Evolution of cortical and thalamus atrophy and disability progression in early relapsing-remitting MS during 5 years. AJNR Am J Neuroradiol 2013;34:1931-9.

7 Zivadinov R, Havrdova E, Bergsland N, et al. Thalamic atrophy is associated with development of clinically definite multiple sclerosis. Radiology 2013;268:831-41.

8 Fisher E, Rudick RA, Simon JH, et al. Eight-year follow-up study of brain atrophy in patients with MS. Neurology 2002;59:1412-20.

9 Fisniku LK, Chard DT, Jackson JS, et al. Gray matter atrophy is related to long-term disability in multiple sclerosis. Ann Neurol 2008:64:247-54. 
10 Horakova D, Dwyer MG, Havrdova E, et al. Gray matter atrophy and disability progression in patients with early relapsing-remitting multiple sclerosis: a 5-year longitudinal study. J Neurol Sci 2009;282:112-19.

11 Rudick RA, Lee JC, Nakamura K, et al. Gray matter atrophy correlates with MS disability progression measured with MSFC but not EDSS. J Neurol SCi 2009;282:106-11.

12 Sastre-Garriga J, Ingle GT, Chard DT, et al. Grey and white matter volume changes in early primary progressive multiple sclerosis: a longitudinal study. Brain 2005; 128:1454-60

13 Dalton CM, Chard DT, Davies GR, et al. Early development of multiple sclerosis is associated with progressive grey matter atrophy in patients presenting with clinically isolated syndromes. Brain 2004;127:1101-7.

14 Bergsland N, Horakova D, Dwyer MG, et al. Subcortical and cortical gray matter atrophy in a large sample of patients with clinically isolated syndrome and early relapsing-remitting multiple sclerosis. AJNR. Am J Neuroradiol 2012;33:1573-8.

15 Calabrese $M$, Rinaldi $F$, Mattisi I, et al. The predictive value of gray matter atrophy in clinically isolated syndromes. Neurology 2011;77:257-63.

16 Henry RG, Shieh M, Okuda DT, et al. Regional Grey Matter Atrophy in Clinically Isolated Syndromes at Presentation. J Neurol Neurosurg Psychiatry 2008;79:1236-44.

17 Fisher E, Lee JC, Nakamura K, et al. Gray matter atrophy in multiple sclerosis: a longitudinal study. Ann Neurol 2008;64:255-65.

18 Neema M, Arora A, Healy BC, et al. Deep gray matter involvement on brain MRI scans is associated with clinical progression in multiple sclerosis. J Neuroimaging 2009;19:3-8.

19 Lukas C, Minneboo A, de Groot V, et al. Early central atrophy rate predicts 5 year clinical outcome in multiple sclerosis. J Neurol Neurosurg Psychiatry 2010;81:1351-6.

20 Roosendaal SD, Bendfeldt K, Vrenken H, et al. Grey matter volume in a large cohort of MS patients: relation to MRI parameters and disability. Mult Scler 2011:17:1098-106.

21 Poser CM, Paty DW, Scheinberg L, et al. New diagnostic criteria for multiple sclerosis: guidelines for research protocols. Ann Neurol 1983;13:227-31.

22 Kurtzke JF. Rating neurologic impairment in multiple sclerosis: an expanded disability status scale (EDSS). Neurology 1983;33:1444-52.

23 Jenkinson $M$, Bannister $\mathrm{P}$, Brady $\mathrm{M}$, et al. Improved optimization for the robust and accurate linear registration and motion correction of brain images. Neurolmage 2002;17:825-41.

24 Zivadinov R, Rudick RA, De Masi R, et al. Effects of IV methylprednisolone on brain atrophy in relapsing-remitting MS. Neurology 2001;57:1239-47.

25 Zivadinov R, Heininen-Brown M, Schirda CV, et al. Abnormal subcortical deep-gray matter susceptibility-weighted imaging filtered phase measurements in patients with multiple sclerosis: a case-control study. Neurolmage 2012;59:331-9.

26 Smith SM, Zhang $Y$, Jenkinson $M$, et al. Accurate, robust, and automated longitudinal and cross-sectional brain change analysis. Neurolmage 2002:17:479-89.

27 Dwyer MG, Bergsland N, Zivadinov R. Improved longitudinal gray and white matter atrophy assessment via application of a 4-dimensional hidden Markov random field model. Neurolmage 2013.
28 Zhang Y, Brady M, Smith S. Segmentation of brain MR images through a hidden Markov random field model and the expectation-maximization algorithm. IEEE Trans Med Imaging 2001:20:45-57.

29 Patenaude B, Smith SM, Kennedy DN, et al. A Bayesian model of shape and appearance for subcortical brain segmentation. Neurolmage 2011;56:907-22.

30 Zivadinov R, Reder A, Filippi M, et al. Mechanisms of action of disease-modifying agents and brain volume changes in multiple sclerosis. Neurology 2008; 71:136-44

31 Minagar A, Barnett MH, Benedict $\mathrm{RH}$, et al. The thalamus and multiple sclerosis: modern views on pathologic, imaging, and clinical aspects. Neurology 2013;80:210-9

32 Pirko I, Lucchinetti CF, Sriram S, et al. Gray matter involvement in multiple sclerosis. Neurology 2007;68:634-42.

33 De Stefano N, Matthews PM, Filippi M, et al. Evidence of early cortical atrophy in MS: relevance to white matter changes and disability. Neurology 2003; 60:1157-62.

34 Tiberio M, Chard DT, Altmann DR, et al. Gray and white matter volume changes in early RRMS: a 2-year longitudinal study. Neurology 2005;64:1001-7.

35 Ramasamy DP, Benedict RH, Cox JL, et al. Extent of cerebellum, subcortical and cortical atrophy in patients with MS: a case-control study. J Neurol Sci 2009;282:47-54.

36 Chard DT, Griffin CM, Parker GJ, et al. Brain atrophy in clinically early relapsing-remitting multiple sclerosis. Brain 2002;125:327-37.

37 Ge Y, Grossman RI, Udupa JK, et al. Brain atrophy in relapsing-remitting multiple sclerosis: fractional volumetric analysis of gray matter and white matter. Radiology 2001;220:606-10.

38 Gelineau-Morel R, Tomassini V, Jenkinson $M$, et al. The effect of hypointense white matter lesions on automated gray matter segmentation in multiple sclerosis. Hum Brain Mapp 2012;33:2802-14

39 Horakova D, Cox JL, Havrdova E, et al. Evolution of different MRI measures in patients with active relapsing-remitting multiple sclerosis over 2 and 5 years: a case-control study. J Neurol Neurosurg Psychiatry 2008;79:407-14.

40 Zivadinov R, Pirko I. Advances in understanding gray matter pathology in multiple sclerosis: Are we ready to redefine disease pathogenesis? BMC Neurol 2012;12:9.

41 Bermel RA, Bakshi R. The measurement and clinical relevance of brain atrophy in multiple sclerosis. Lancet Neurol 2006;5:158-70.

42 Zivadinov R, Bakshi R. Central nervous system atrophy and clinical status in multiple sclerosis. J Neuroimaging 2004;14:27S-35S.

43 Polman CH, Reingold SC, Banwell B, et al. Diagnostic criteria for multiple sclerosis: 2010 revisions to the McDonald criteria. Ann Neurol 2011;69:292-302.

44 Caramanos Z, Francis SJ, Narayanan S, et al. Large, nonplateauing relationship between clinical disability and cerebral white matter lesion load in patients with multiple sclerosis. Arch Neurol 2012;69:89-95.

45 Popescu V, Agosta F, Hulst HE, et al. Brain atrophy and lesion load predict long term disability in multiple sclerosis. J Neurol Neurosurg Psychiatry 2013;84:1082-91.

46 Bendfeldt $\mathrm{K}$, Hofstetter L, Kuster $\mathrm{P}$, et al. Longitudinal gray matter changes in multiple sclerosis-differential scanner and overall disease-related effects. Hum Brain Mapp 2012;33:1225-45 